\title{
La métrologie du risque radon dans les habitations et l'environnement*
}

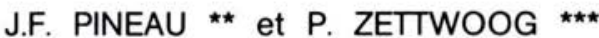

\section{RÉSUMÉ}

Après un bref rappel du phénomène radon, le document ci-après présente les principales grandeurs à mesurer ainsi que les unités correspondantes.

Le second chapitre traite des objectifs métrologiques et tente de définir le profil des demandeurs et le type de besoins à satisfaire.

Les méthodes et appareils de mesure du radon et de ses descendants sont décrites dans le chapitre 3 .

Le dernier chapitre traite enfin de l'application de la métrologie du radon et de ses descendants à la mesure des sources, des ambiances domestiques et des expositions individuelles.

\section{ABSTRACT}

Following a short review of the radon phenomenon, the main quantities to be measured and the corresponding units are presented. The metrologic objectives are specified together with an attempt to defining the applicants' profiles and the types of requirements to be satisfied. The methods and instruments for the measurements of radon and its daughters are described. Finally, the application of the metrology of radon and its daughter products to the measurement of sources, indoor air and individual exposures is presented.

\section{INTRODUCTION}

Le radon est un gaz rare dont il existe trois isotopes naturels, ${ }^{219} \mathrm{Rn}$, ${ }^{220} \mathrm{Rn},{ }^{222} \mathrm{Rn}$, respectivement descendant de ${ }^{223} \mathrm{Ra},{ }^{224} \mathrm{Ra},{ }^{226} \mathrm{Ra}$, et appartenant aux chaînes de ${ }^{235} \mathrm{U},{ }^{238} \mathrm{U},{ }^{232} \mathrm{Th}$. Le risque sanitaire du radon est, en fait, lié aux descendants à vie courte de ses isotopes. Le risque principal en valeur relative est celui dû aux descendants du ${ }^{222} \mathrm{Rn}$. Le risque dû aux descendants du ${ }^{220} \mathrm{Rn}$ est de l'ordre de $10 \%$ du précédent. Mais les activités volumiques dans l'atmosphère de ${ }^{220} \mathrm{Rn}$ et de ${ }^{222} \mathrm{Rn}$ sont souvent du même ordre de grandeur. Le risque dû aux descendants du ${ }^{219} \mathrm{Rn}$ est négligeable en valeur relative, ainsi que son activité volumique, sauf lorsque le temps de transit entre la source et l'appareil de mesure est de quelques secondes seulement.

* Communication présentée lors de la réunion SFRP "Exposition au radon dans les habitations: aspects techniques et sanitaires", Paris, 10-11 janvier 1989.

* Commissariat à l'énergie atomique, Institut de protection et de sûreté nucléaire, CRPM/DIA, Fanay, 87240 Saint-Sylvestre.

*** Commissariat à l'énergie atomique, Institut de protection et de sûreté nucléaire, DPT/SPIN, BP 6, 92265 Fontenay-aux-Roses Cedex. 
Les quantités de ces radionucléides mises en jeu étant très faibles, il est commode de les caractériser par le nombre d'atomes de chacun d'eux. Mais ce qu'on mesure, c'est la vitesse de décroissance de ces atomes, c'est-àdire leur activité. Entre le nombre d'atomes $\mathrm{N}$ et l'activité $\mathrm{A}$, il y a la relation:

$$
A=\lambda N
$$

Pour les trois isotopes $219,220,222, \lambda$ vaut respectivement, en $\mathrm{s}^{-1}: 0,12$; $1,26.10^{-2} ; 2,1.10^{-6}$.

Les périodes radioactives se calculent par $T=0,7 / \lambda$.

$$
{ }^{219} \mathrm{~T}=4 \mathrm{~s} \quad{ }^{220} \mathrm{~T}=56,6 \mathrm{~s} \quad{ }^{222} \mathrm{~T}=3,8 \mathrm{j} .
$$

Une activité $\mathrm{A}$ de $1 \mathrm{~Bq}$, soit une désintégration par seconde, correspond à: 476000 atomes de ${ }^{222} \mathrm{Rn} ; 79$ atomes de ${ }^{220} \mathrm{Rn} ; 7$ atomes de ${ }^{219} \mathrm{Rn}$.

La complexité de la physique du risque radon oblige ceux qui veulent le mesurer à commencer par comprendre quels sont les phénomènes qui interviennent. De là, on déduit les grandeurs à mesurer aux différents stades d'avancement des processus d'exposition des personnes.

\section{Phénoménologie; GRANDEuRs À mesurer; Unités}

\subsection{Les sources}

Le radon que l'on trouve dans une maison résulte de la décroissance des isotopes du radium qui sont toujours présents, en plus ou moins grande quantité, dans les matériaux de construction ainsi que dans les roches du soubassement et du sous-sol. II est ensuite transporté par diffusion et souvent aussi par convection dans l'air ambiant intérieur.

Les matériaux de construction représentent une source de radon proportionnelle au produit d'un coefficient sans dimension compris entre 0 et 1 , différent selon les isotopes, et de l'activité totale de l'isotope du radium correspondant contenue dans la maison. Ce coefficient varie selon les minéraux porteurs d'activité, la porosité et l'épaisseur des murs. La source est une grandeur physique qui s'exprime en atomes par seconde.

Dans les matériaux de construction usuels, l'activité des isotopes du radium parents de ${ }^{220} \mathrm{Rn}$ et ${ }^{222} \mathrm{Rn}$ est de l'ordre de 10 à $100 \mathrm{~Bq} \cdot \mathrm{kg}^{-1}, 5$ à $30 \%$ du ${ }^{222} \mathrm{Rn}$ formé dans les murs parvient dans l'air ambiant intérieur. On mesure parfois directement les flux de radon aux parois ou au sol. Ils s'expriment en atomes par seconde et par mètre carré.

Le sous-sol de la maison peut être le siège d'une remontée d'air en provenance des roches du soubassement et du milieu géologique lui-même. Ce phénomène se produit sous l'action de faibles différences de pression induites par le vent ou le chauffage. Cet air contient toujours de très fortes concentrations de ${ }^{222} \mathrm{Rn}$. Dans l'eau des nappes phréatiques, on trouve 
les mêmes concentrations, si bien que l'utilisation de ces eaux pour les douches où il se dégage est une autre façon d'apporter du radon. Des apports par le gaz naturel, où le radon encore une fois provient du milieu géologique, sont cités dans la littérature.

Le radon émis à la surface de la terre se dilue dans l'atmosphère. De là, il pénètre dans la maison. Connaissant le débit d'aérage de la maison et les concentrations en radon (atomes par mètre cube), ou les activités volumiques becquerels par mètre cube, on en déduit les quantités rentrantes.

\subsection{Formation de la pollution dans l'air ambiant intérieur}

La pollution de l'air ambiant est caractérisée par sa concentration en chacun des isotopes du radon et de leurs descendants à vie courte (atomes par mètre cube). La compréhension des phénomènes de formation des descendants successifs est facilitée si l'on raisonne atome par atome, car dans une même chaîne le nombre total des atomes est conservé. Pour décrire les phénomènes, on introduit, en plus des temps de décroissance radioactive de chaque radionucléide, le temps de renouvellement de l'air de la pièce, $\mathrm{Tr}$, le temps d'homogénéisation (qui est de quelques minutes), et le temps de placage aux parois des aérosols porteurs des descendants à vie courte, de l'ordre de l'heure.

Quand la pièce a ses fenêtres largement ouvertes, il n'y a pas de différence de pollution entre l'intérieur et l'extérieur. On ne peut parler de pollution "domestique" que si la pièce est à peu près fermée. On a alors des temps de renouvellement de l'air allant de un quart d'heure à deux heures.

Cas $d u{ }^{222} R n$ - La perte pour décroissance n'est que de 0,75\% par heure et on peut la négliger. La concentration en ${ }^{222} \mathrm{Rn},(\mathrm{Ci})_{222}$, est donnée par:

$$
(\mathrm{Ci})_{222}=(\mathrm{Cex})_{222}+\frac{(\mathrm{Si})_{222} \cdot \mathrm{Tr}}{\mathrm{V}} \text { avec : }
$$

$(\mathrm{Ci})_{222}$ : concentration en ${ }^{222} \mathrm{Rn}$ dans la pièce (atome $\mathrm{m}^{-3}$ );

$(\mathrm{Cex})_{222}$ : concentration en ${ }^{222} \mathrm{Rn}$ dans l'air rentrant dans la pièce (atome $\cdot \mathrm{m}^{-3}$ );

$(\mathrm{Si})_{222}$ : source interne de radon dans la pièce (atome. $\mathrm{s}^{-1}$ );

$\operatorname{Tr} \quad$ : temps de tenouvellement dans l'air (s);

$\mathrm{V} \quad$ : volume de la pièce $\left(\mathrm{m}^{3}\right)$;

$\mathrm{V} / \mathrm{Tr}$ est le débit de ventilation de la pièce $\left(\mathrm{m}^{3} \cdot \mathrm{s}^{-1}\right)$.

Les radioprotectionnistes ont l'habitude de raisonner en activité (becquerel). On passe des concentrations $\mathrm{C}$ (atome $\mathrm{m}^{-3}$ ) aux activités volumiques $\mathrm{A}_{v}\left(\mathrm{~Bq} \cdot \mathrm{m}^{-3}\right)$, par $\mathrm{A}_{\mathrm{v}}=\lambda$.C. Ici $\lambda=2,1 \cdot 10^{-6}$. 
Les descendants à vie courte du ${ }^{222} \mathrm{Rn}$ ont des périodes radioactives comprises entre le temps d'homogénéisation et le temps de renouvellement ou le temps de placage. Ceci veut dire que, d'une part, leur distribution dans une pièce est à peu près homogène, sauf au voisinage immédiat des entrées d'air frais, et que, d'autre part, ils n'auront pas le temps d'être en équilibre radioactif avec les radionucléides parents.

Ce qui compte sur le plan sanitaire c'est l'énergie des particules alpha que les aérosols inhalés et porteurs de descendants à vie courte vont pouvoir potentiellement délivrer aux tissus pulmonaires. On introduit l'énergie alpha potentielle volumique, $\mathrm{EAP}_{\mathrm{v}}$, qui s'exprime légalement en joules par mètre cube. Entre l'EAP $v$ que l'on $a$, et celle que l'on aurait si tous les descendants à vie courte étaient à l'équilibre radioactif avec ${ }^{222} \mathrm{Rn}$, il y a un rapport que l'on appelle le facteur d'équilibre équivalent $(F)$. F croît avec le temps de renouvellement et avec le temps de placage.

Souvent, on introduit par commodité l'activité volumique d'équilibre équivalente $\left(A_{v} E E\right)$ du ${ }^{222} R n\left(B q \cdot m^{-3}\right)$. Si $A_{v}$ est l'activité volumique de ${ }^{222} R n$ $\left(B q \cdot m^{-3}\right)$, on a par définition $A_{v} E E=F A_{v}$. C'est une manière alternative de caractériser $E A P_{v}$. On a les équivalences: $100 \mathrm{~Bq} \cdot \mathrm{m}^{-3}\left(A_{v} E E\right)=$ $0,554 \mu \mathrm{J} . \mathrm{m}^{-3}$.

$$
\text { On a donc }\left(\mathrm{EAP}_{\mathrm{v}}\right)_{222}=5,54 \cdot 10^{-3} \cdot \mathrm{F} \cdot\left(\mathrm{A}_{\mathrm{v}}\right)_{222}\left(\mu \mathrm{J} \cdot \mathrm{m}^{-3}\right)
$$

Cas̀ $d u{ }^{220} R n$ - La période radioactive est de l'ordre de grandeur du temps d'homogénéisation; elle est faible vis-à-vis du temps de renouvellement. La concentration intérieure en ${ }^{220} \mathrm{Rn}$, qui peut ne pas être homogène dans la pièce, est donnée par:

$$
(\mathrm{Ci})_{220}=\frac{(\mathrm{Si})_{220}+(\mathrm{Sex})_{220}}{\mathrm{~V} .0,7}(\mathrm{~T})_{220} \text { avec: }
$$

$(\mathrm{Ci})_{220}$ : concentration intérieure en ${ }^{220} \mathrm{Rn}$ (atome. $\mathrm{m}^{-3}$ );

$(\mathrm{T})_{220}$ : période radioactive du ${ }^{220} \mathrm{Rn}(56,6 \mathrm{~s})$;

(Si) 220 : source intérieure du ${ }^{220} \mathrm{Rn}$ (atome. $\mathrm{s}^{-1}$ );

$(\text { Sex })_{220}$ : source extérieure équivalente (apport par l'air rentrant) (atome. $\left.\mathrm{s}^{-1}\right)$;

V : volume de la pièce $\left(\mathrm{m}^{3}\right)$;

Par désintégration, le ${ }^{220} \mathrm{Rn}$ se transforme rapidement en deux étapes en ${ }^{212} \mathrm{~Pb}$, dont le temps de décroissance, $10,6 \mathrm{~h}$, est grand vis-à-vis de $\mathrm{Tr}$. On peut dire que tous les atomes de ${ }^{220} \mathrm{Rn}$ apportés par la source deviennent, nombre pour nombre, des atomes de ${ }^{212} \mathrm{~Pb}$. La concentration intérieure de ${ }^{212} \mathrm{~Pb}$ est donc, en supposant que les atomes de ${ }^{212} \mathrm{~Pb}$ de l'extérieur sont filtrés:

$$
(\mathrm{Ci})_{212}=\frac{(\mathrm{Si})_{220}+(\mathrm{Sex})_{220} \cdot \mathrm{Tr}}{\mathrm{V}}\left(\text { atome } \cdot \mathrm{m}^{-3}\right)
$$


A chacun de ces atomes de ${ }^{212} \mathrm{~Pb}$ correspond une énergie potentielle alpha $\left(E A P_{v}\right)$ de $1,35.10^{-12} \mathrm{~J}$ par atome. On a donc:
$\left(\mathrm{EAP}_{\mathrm{v}}\right)_{220}=1,35 \cdot 10^{-6} \mathrm{x}$
$(\mathrm{Ci})_{212}$
$\left(\mu \mathrm{J} \cdot \mathrm{m}^{-3}\right)$

Entre les concentrations $\mathrm{Ci}$ des deux isotopes 220 et 222 et en supposant négligeables $(\mathrm{Cex})_{222}$ et $(\operatorname{Sex})_{220}$, on a le rapport:

$$
\frac{(\mathrm{Ci})_{220}}{(\mathrm{Ci})_{222}}=\frac{(\mathrm{Si})_{220}}{(\mathrm{Si})_{222}} \cdot \frac{(\mathrm{T})_{220}}{\mathrm{~T} \cdot 0,7}
$$

Dans une chambre d'ionisation à circulation, c'est le rapport des activités volumiques (Avi) qui est mesurable. On a:

$$
\frac{(\text { Avi })_{220}}{(\text { Avi })_{222}}=\frac{(\mathrm{Ci})_{220}}{(\mathrm{Ci})_{222}} \times \frac{(\mathrm{T})_{222}}{(\mathrm{Si})_{220}}=\frac{(\mathrm{Si})_{220}}{(\mathrm{Si})_{222}} \cdot \frac{(\mathrm{T})_{222}}{\mathrm{~T} .0 .7} \text { avec }:(\mathrm{T})_{222}=3,8 \mathrm{j}
$$

Ce rapport est d'autant plus élevé que la pièce est bien ventilée. Le rapport des termes sources est proportionnel au rapport des activités en ${ }^{232} \mathrm{Th}$ et ${ }^{238} \mathrm{U}$ des matériaux de construction, ceci lorsque le radon ne provient pas du sol. Des valeurs de l'ordre de l'unité sont possibles pour ce rapport pour les faibles concentrations en ${ }^{222} \mathrm{Rn}$.

En ce qui concerne le rapport des $\mathrm{EAP}_{\mathrm{v}}$, on a, de ce qui précède (relations (1) et (2):

$$
\frac{\left(\mathrm{EAP}_{\mathrm{v}}\right)_{220}}{(\mathrm{EAP})_{222}}=\frac{1,16.10^{2}}{\mathrm{~F}} \cdot \frac{(\mathrm{Si})_{220}}{(\mathrm{Si})_{222}}
$$

Pour des activités égales de ${ }^{232} \mathrm{Th}$ et ${ }^{238} \mathrm{U}$ dans les matériaux, on a en général $(\mathrm{Si})_{220} /(\mathrm{Si})_{222}<<1$, car le radon 220 disparaît par décroissance avant de sortir des murs et peut être arrêté par un revêtement mural ou par le plâtre.

Cas $d u{ }^{219} R n$ - Cet isotope n'intervient pas en pratique dans les mesures.

\subsection{Exposition individuelle et énergie inhalée}

Le risque est dû à ce que les personnes sont exposées à cet air pollué et l'inhalent. Dans le cadre des hypothèses habituelles en radioprotection d'absence de seuil et de linéarité entre dose d'irradiation de bas niveau et d'effets sanitaires probabilistes, l'effet sanitaire est proportionnel au produit de l'EAP $\mathrm{v}_{\mathrm{v}}$ par le temps d'exposition, plus exactement à l'intégrale de EAP $_{\mathrm{v}}$ sur la durée du temps de présence des individus dans la maison. L'exposition (Ex) est exprimée en $\mathrm{mJ} \cdot \mathrm{m}^{-3} \cdot \mathrm{h}$. On a les équivalences: $1 \mathrm{~mJ} \cdot \mathrm{m}^{-3} \cdot \mathrm{h}=$ 0,28 WLM. 
WLM est une unité d'exposition anglo-saxonne non légale, encore utilisée dans les enquêtes épidémiologiques. Les Communautés européennes introduisent dans leurs directives la quantité annuelle Qi d'énergie alpha potentielle inhalée. C'est le produit de Ex par le débit d'inhalation. Pour la première fois, on introduit à ce stade un paramètre biologique, qui varie selon l'âge et l'occupation. Tant pour ${ }^{220} \mathrm{Rn}$ que pour ${ }^{222} \mathrm{Rn}$, Qi s'exprime en $\mathrm{mJ} . \mathrm{an}^{-1}$. Les coefficients de risque associés aux énergies inhalées étant différents pour le radon 220 et pour le radon 222 (facteur 3 en plus sévère pour 222 que pour 220), il convient de les évaluer séparément. Pour l'individu standard de la population générale, on admet un débit moyen journalier d'inhalation de $0,8 \mathrm{~m}^{3} \cdot \mathrm{h}^{-1}$. Les quantités inhalées dans les maisons sont de l'ordre du $\mathrm{mJ} . \mathrm{an}^{-1}$.

\subsection{Résumé}

Les grandeurs à mesurer sont:

- les sources de radon 220 et 222 , exprimées en atome. $\mathrm{s}^{-1}$;

- pour l'air ambiant:

- les concentrations en radon 220 et 222 (atome. $\mathrm{m}^{-3}$ ),

- ou leur activité volumique $\left(\mathrm{Bq} \cdot \mathrm{m}^{-3}\right)$,

- ou l'énergie alpha potentielle de leurs descendants $\left(\mu \mathrm{J} . \mathrm{m}^{-3}\right)$;

- pour l'individu, l'exposition à l'énergie alpha potentielle des descendants des radon 220 et 222 en $\mathrm{mJ} . \mathrm{m}^{-3} . \mathrm{h}$.

\section{OBJECTIFS MÉtROLOGIQUES ET DEMANDEURS}

D'une part de ce qui précède, d'autre part des recommandations de la CIPR, exprimées notamment dans ses publications 39 et 50 , un certain nombre d'objectifs métrologiques se dégagent, chacun d'eux étant à associer à des demandeurs dont les besoins doivent être bien identifiés: la qualité de la mesure en dépend.

\subsection{Objectif de mise en alerte sur les maisons}

Un objectif fondamental est celui du père de famille qui se demande s'il y a du radon dans sa maison. Pour un physicien la question n'a peut être pas de sens (il y en a toujours !), mais pour un père de famille elle est très pertinente et sans ambiguitté: la CIPR recommande de n'engager aucune

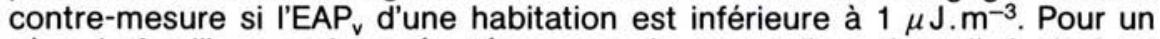
père de famille sera donc réputée sans radon une telle maison. II s'agit donc de mesurer EAP $_{\mathrm{v}}$ dans l'habitation. La mesure devant être précise aux environs de $\mathrm{EAP}_{\mathrm{v}}=1 \mu \mathrm{J} \cdot \mathrm{m}^{-3}$, le mieux est de le faire dans une pièce fermée depuis au moins $12 \mathrm{~h}$, et il est judicieux de choisir les chambres à coucher des parents et des enfants. Des contrôles dans la cave ou le sous-sol ne seront pas inutiles. 
L'idéal dans cet objectif de mise en alerte est que la mesure soit faite instantanément. Des appareils existent qui permettent la mesure instantanée, mais ils doivent être utilisés par des spécialistes.

Par contre, il y a sur le marché des appareils autonomes que tout le monde peut mettre en œuvre, seul chez lui, pour un prix modique, à l'aide de la notice, avec un résultat obtenu d'autant plus vite que les niveaux sont forts, en fait en un temps de quelques heures à 1 ou 2 jours suivant le niveau de EAP $_{\mathrm{v}}$. Au niveau $1 \mu \mathrm{J} . \mathrm{m}^{-3}$ le résultat est obtenu en 1 jour.

Si le résultat vérifié $\mathrm{EAP}_{\mathrm{v}}<1 \mu \mathrm{J} \cdot \mathrm{m}^{-3}$ dans toutes ses pièces d'habitation, il ne va pas plus loin. On notera que cet objectif est aussi celui de qui va acheter une maison et par conséquent, de qui veut la vendre et, par extension, celui des professionnels de l'immobilier.

\subsection{Mesure des niveaux de risque individuels (familiaux)}

Un deuxième objectif fondamental apparaît si le père de famille trouve que dans sa maison $\mathrm{EAP}_{\mathrm{v}}>1 \mu \mathrm{J} . \mathrm{m}^{-3}$. II lui est alors conseillé de faire une analyse plus approfondie des risques encourus par lui et les siens. II doit s'adresser à un laboratoire spécialisé qui mettra en place ou lui livrera le matériel nécessaire à ce deuxième objectif métrologique, à savoir la mesure à un coût accessible des expositions individuelles des membres de sa famille. Le résultat est d'autant plus sûr et significatif que la mesure est de longue durée, 3 mois, par exemple. Cela permet de lisser les variations temporelles diurnes, hebdomadaires, saisonnières. Un mois est un minimum. II faut se rappeler que, sauf valeur d'EAP $v$ exceptionnellement élevée, on a le temps devant soi pour prendre des contre-mesures; le danger, s'il existe, n'est pas immédiat.

Différents matériels existent sur le marché qui permettent la mesure des expositions ambiantes dans les pièces où les membres de la famille passent le plus de temps. On remonte aux expositions individuelles strictes en tenant compte des temps de présence.

\subsection{Diagnostics techniques}

Un troisième objectif métrologique est associé à cette analyse. C'est le diagnostic technique. Si des contre-mesures sont à prendre, quelles sontelles? La réponse passe par un diagnostic sur les causes de la pollution ambiante et sur les coûts des remèdes envisageables. Tous les éléments sont alors réunis pour qu'une analyse des coûts et des bénéfices des remèdes soit effectuée. Le père de famille doit alors s'adresser à un technicien radon qui possède des appareils de mesure instantanée des isotopes du radon et de ses descendants, capables de mesurer les sources et les flux, les concentrations, les débits de ventilation, les temps d'homogénéisation et de placage, les facteurs d'équilibre des descendants à vie courte.

Avec le même matériel, il pourra vérifier l'efficacité d'éventuelles contremesures décidées par son client. 
Ce technicien travaillera aussi pour les entrepreneurs chargés de constructions nouvelles. II les aidera à atteindre l'objectif $\mathrm{EAP}_{\mathrm{v}}<0,5 \mu \mathrm{J} \cdot \mathrm{m}^{-3}$ pour les maisons à construire et à démontrer qu'il a été obtenu.

Ces trois premiers objectifs relèvent des intérêts privés et les résultats de mesure, notamment dans les deux premiers objectifs, sont en principe confidentiels. Toutefois, ils peuvent concerner des écoles, des internats et maisons de retraite, des hôpitaux de longue durée.

\subsection{Connaissance de la pollution extérieure}

II existe des objectifs où l'intérêt est plus général: c'est ainsi qu'il est de l'intérêt des collectivités de savoir quels sont les niveaux de radon dans l'atmosphère libre. Ces niveaux varient considérablement entre la nuit et le jour, et suivant les régions. L'essentiel de l'exposition domestique peut, parfois, être causé par le radon extérieur. Les contre-mesures qui sont engagées sont alors de faiblè efficacité, et il vaut mieux le savoir tout de suite. Un quatrième objectif est donc la connaissance des variations des niveaux de radon 220 et 222 et l'échelle locale, celle du lotissement, du village, du quartier, de la vallée.

II faut des matériels capables de mesurer en continu, pouvant fonctionner à poste fixe sur des durées assez longues, ou susceptibles d'être embarqués dans des laboratoires mobiles, de façon à dresser la carte de la pollution radon. Les demandeurs sont ici des responsables municipaux ou associatifs, à l'échelle locale, ou des autorités départementales, à l'échelle régionale.

\subsection{Campagne de "zonation" des risques}

Un cinquième objectif d'intérêt général peut être encore dégagé. II s'agit d'effectuer des campagnes d'investigation à l'échelle régionale, devant permettre le repérage des zones du territoire présentant des anomalies de flux de radon, qui peuvent correspondre à des failles géologiques actives à l'échelle régionale, ou à des teneurs fortes, voire à des indices de minéralisation, en uranium ou en thorium. II faut aussi repérer les zones où les maisons sont construites en matériaux contenant des teneurs fortes en $U$ et en Th. Ce type d'informations concerne les personnes responsables de l'aménagement du territoire et les promoteurs immobiliers. Elle intéresse également les familles, car ces informations font apparaître les zones à risque potentiel élevé, donc celles où les habitants seraient avisés de procéder à des vérifications selon l'objectif 1 .

Les matériels nécessaires sont les mêmes que pour l'objectif 4 , avec, en plus, des moyens de mesure de l'irradiation externe gamma des matériaux et de mesure des flux de radon dans les sols. Ces matériels sont avantageusement mis en œuvre dans des laboratoires mobiles de cartographie, comportant les moyens ad hoc de collecte et de traitement d'informations géocodées. 


\section{MÉtHODES UTILISÉES POUR LA MESURE DES ISOTOPES DU RADON \\ ET DE LEURS DESCENDANTS DANS L'AIR; MATÉRIELS DÉRIVÉS}

\subsection{Rappel}

Les différentes méthodes de mesure du radon et de ses descendants sont toutes basées sur la détection des particules dont l'émission accompagne les désintégrations radioactives successives des isotopes du radon et de leurs descendants.

L'indicateur de risque principal étant l'exposition à l'énergie alpha potentielle des descendants du radon, toutes les méthodes utilisées doivent, en fin de compte, permettre d'estimer, soit individuellement, soit globalement, les activités volumiques des descendants émetteurs alpha à vie courte dans l'air.

Certains descendants, émetteurs alpha potentiels, sont aussi émetteurs bêta et gamma. On peut donc aussi se servir de ces rayonnements pour assurer leur détection et leur mesure.

D'autre part, les périodes de ces descendants étant très courtes, on devra, lors de l'analyse, tenir compte de la décroissance radioactive pouvant avoir eu lieu entre le prélèvement et la mesure ou durant la mesure elle-même.

On rappelle, dans le tableau ci-dessous, les principales caractéristiques des radionucléides concernés.

\begin{tabular}{|c|c|c|c|c|c|}
\hline \multicolumn{3}{|c|}{${ }^{238} \mathrm{U}$} & \multicolumn{3}{|c|}{${ }^{235} \mathrm{U}$} \\
\hline Eléments & Période & $\begin{array}{l}\text { Principaux } \\
\text { rayonnements } \\
(\mathrm{keV})\end{array}$ & Eléments & Période & $\begin{array}{l}\text { Principaux } \\
\text { rayonnements } \\
(\mathrm{keV})\end{array}$ \\
\hline $\begin{array}{l}{ }^{222} \mathrm{Rn} \\
218 \mathrm{Po} \\
214 \mathrm{~Pb} \\
214 \mathrm{Bi} \\
{ }^{214} \mathrm{Po}\end{array}$ & $\begin{array}{l}3,8 \mathrm{j} \\
3,11 \mathrm{~min} \\
26,8 \mathrm{~min} \\
19,5 \mathrm{~min} \\
10^{-3} \mathrm{~s}\end{array}$ & $\begin{array}{c}5490 \text { alpha } \\
6000 \text { alpha } \\
\text { béta } \\
\text { bêta } 609 \text { alpha } \\
7700 \text { alpha }\end{array}$ & $\begin{array}{l}{ }^{219} \mathrm{Rn} \\
215 \mathrm{Po} \\
211 \mathrm{~Pb} \\
211 \mathrm{Bi} \\
211 \mathrm{Po} \\
{ }^{207} \mathrm{Tl}\end{array}$ & $\begin{array}{l}4 \mathrm{~s}^{-3} \mathrm{~s} \\
10^{-3} \mathrm{~s} \\
36,1 \mathrm{~min} \\
2,13 \mathrm{~min} \\
0,52 \mathrm{~s} \\
4,8 \mathrm{~min}\end{array}$ & $\begin{array}{c}5770 \text { alpha } \\
7380 \text { alpha } \\
\text { bêta } \\
6620 \text { alpha } \\
7450 \text { alpha } \\
\text { bêta }\end{array}$ \\
\hline
\end{tabular}

\begin{tabular}{|l|l|c|}
\hline & \multicolumn{2}{|c|}{${ }^{232} \mathrm{Th}$} \\
Eléments & Période & $\begin{array}{c}\text { Principaux } \\
\text { rayonnements } \\
\text { (keV) }\end{array}$ \\
\hline${ }^{220} \mathrm{Rn}$ & $55,6 \mathrm{~s}$ & 6290 alpha \\
$216 \mathrm{Po}$ & $0,15 \mathrm{~s}$ & $\begin{array}{c}6780 \text { alpha } \\
\text { bêta }\end{array}$ \\
$212 \mathrm{~Pb}$ & $10,6 \mathrm{~h}$ & 6000 alpha $30 \%$ \\
$212 \mathrm{Bi}$ & $61 \mathrm{~min}$ & 8780 alpha $60 \%$ \\
$212 \mathrm{Po}$ & $3.10^{-7} \mathrm{~s}$ & 870 \\
\hline
\end{tabular}




\subsection{Méthodes utilisant la scintillation du sulfure de zinc ou de l'air}

Elles sont basées sur l'excitation des couches électroniques de molécules de sulfure de zinc activé à l'argent ( $\mathrm{ZnS}(\mathrm{Ag})$ ) sous l'effet des particules alpha du radon et de ses descendants. Le parcours des alpha dans la matière étant très faible (quelques dizaines de micromètres), la particule cède donc toute son énergie au milieu ambiant; cette énergie se retrouve sous la forme de niveaux excités qui, en revenant au niveau fondamental, émettent des photons. Ces photons, dans le cas du $\mathrm{ZnS}(\mathrm{Ag})$, sont émis vers $450 \mathrm{~nm}$ et peuvent être détectés à l'aide d'un photomultiplicateur. C'est le principe adopté pour les fioles scintillantes (dites fioles de Lucas) et qui sont utilisées pour la mesure du radon gaz.

La mesure par scintillation de l'air repose sur la même technique, les molécules cibles étant, cette fois-ci, l'azote de l'air, et les photons étant émis dans I'UV. L'utilisation de cette technique permet une mesure de la concentration en radon sans perturber le milieu à mesurer. Elle n'est à envisager que pour de fortes concentrations sur des volumes de grande profondeur optique.

La scintillation du ZnS (Ag) peut aussi être utilisée pour la détection des descendants du radon collectés sur un filtre; le filtre est placé au contact d'un disque scintillant au $\mathrm{ZnS}(\mathrm{Ag})$ lui même placé sur un photomultiplicateur relié à une chaîne de comptage.

L'analyse mathématique des comptages permet de calculer l'activité volumique des différents descendants ainsi que l'énergie alpha potentielle résultante.

\subsection{Méthodes utilisant l'ionisation de l'air}

Le libre parcours dans l'air des particules alpha émises par le radon et ses descendants est de l'ordre de quelques centimètres. La particule va donc perdre toute son énergie sur ce parcours et, comme l'énergie moyenne de création d'une paire d'ions dans l'air est de $36 \mathrm{eV}$, on voit que chaque particule alpha créera, lors de son parcours dans l'air, plusieurs dizaines de milliers de paires d'ions. Si l'on applique un champ électrique entre deux électrodes placées dans cet air, il y aura collection des ions sur les électrodes et création d'un courant d'ionisation. La mesure de ce courant, bien que très faible $\left(10^{-14} \mathrm{~A}\right.$ dans des volumes de l'ordre de la dizaine de litres), est possible et la chambre d'ionisation ainsi constituée permet la mesure de l'activité volumique du radon et de ses descendants. Si l'air mis en circulation à travers la chambre est filtrée, le signal sera alors proportionnel à l'activité volumique du radon gaz. Les chambres d'ionisation constituent ainsi un moyen simple pour la mesure en continu de l'activité volumique du radon gaz. Une extrapolation des chambres d'ionisation conduit au montage à compteur dit proportionnel, mettant en jeu des courants beaucoup plus importants. De plus, la combinaison de deux chambres d'ionisation avec un volume de vieillissement permet, en jouant sur la différence entre les périodes des isotopes 220 et 222 du radon, de mesurer ceux-ci séparément et d'extraire le signal dû au rayonnement gamma ambiant. 


\subsection{Méthodes utilisant les détecteurs solides de trace}

De la même façon que dans l'air, le passage d'une particule alpha dans certains solides minéraux ou organiques provoque une ionisation des molécules présentes sur le passage. Du fait de la structure solide, les recombinaisons ioniques, après le passage de la particule, ne sont pas complètes.

Le détecteur est parfois exposé nu mais, en général, les utilisateurs préfèrent imposer une géométrie (le volume de mesure) et limiter l'accès à ce volume aux seuls atomes de radon par l'usage d'un filtre arrêtant les descendants du radon formés à l'extérieur de l'appareil; certains arrivent à éliminer le ${ }^{220} \mathrm{Rn}$ à vie très courte.

Une attaque chimique appropriée sert de révélateur et le matériau utilisé présente alors des traces sous la forme de trous ou de cônes d'attaque, dont le nombre est égal, en première approximation, au nombre de particules alpha ayant pénétré dans le détecteur. Un comptage optique ou électrique de ces traces permet alors, selon la configuration d'utilisation du détecteur, de remonter à la concentration en radon ou en énergie alpha potentielle. Dans certains détecteurs de traces, l'analyse de la géométrie des cônes d'attaque permet théoriquement de remonter à la concentration des isotopes du radon et du polonium. Du fait de leur passivité (pas de pompe ni d'alimentation électrique) et de leur faible coût relatif, ces détecteurs ont été extensivement utilisés pour les campagnes d'investigation du risque radon.

\subsection{Méthodes utilisant la thermoluminescence}

Les matériaux thermoluminescents, comme le détecteur solide de traces, gardent une "trace latente" du passage d'un rayonnement ionisant. Un chauffage ultérieur entraîne une recombinaison ionique accompagnée d'une émission de photons, le nombre de photons émis étant alors proportionnel à la dose délivrée au matériau. L'utilisation de tels matériaux thermoluminescents a été tentée pour la détection des particules alpha. Vu le faible parcours des alpha dans le matériau, seule la couche superficielle est active et le dispositif est très sensible aux défauts de cette surface.

L'utilisation de ces matériaux pour la mesure des rayonnements gamma émis par certains descendants reste, quant à elle, du domaine du laboratoire, les doses délivrées par les descendants du radon dans l'air, en particulier, le ${ }^{214} \mathrm{Bi}$ étant, même pour les concentrations en radon les plus fortes, inférieures d'un ordre de grandeur au rayonnement gamma ambiant.

\subsection{Spectrométrie gamma}

L'utilisation de la spectrométrie gamma pour la mesure du radon s'est développée avec la mise au point des détecteurs passifs utilisant le charbon actif comme élément de rétention du radon. Celui-ci, piégé sur les grains de 
charbon, se désintègre localement et une analyse par spectrométrie gamma des ${ }^{214} \mathrm{~Pb}$ et ${ }^{214} \mathrm{Bi}$ permet, par la connaissance du temps de décroissance du ${ }^{222} \mathrm{Rn}$, le calcul de la concentration moyenne en ${ }^{222} \mathrm{Rn}$ pendant la durée d'exposition du détecteur. Les incertitudes de cette méthode élégante sont liées à la variabilité du coefficient d'absorption du radon 222 par le charbon en fonction de facteurs environnementaux tels que l'humidité ou certaines vapeurs organiques, ainsi que son vieillissement.

La spectrométrie gamma, appliquée aux raies des ${ }^{214} \mathrm{~Pb}$ et ${ }^{214} \mathrm{Bi}$ et complétée d'un calcul mathématique permet aussi l'analyse d'un prélèvement de descendants du radon sur un filtre. Cependant, la nécessité d'utiliser un détecteur au germanium refroidi à l'azote liquide implique la mise en œuvre par un laboratoire spécialisé; cette technique est très utilisée aux États-Unis.

\subsection{Spectrométrie alpha}

La spectrométrie alpha (le comptage alpha peut être considéré comme une spectrométrie à 1 seul canal) utilise un détecteur silicium qui délivre des impulsions d'amplitude proportionnelle à l'énergie des particules alpha incidentes. Un choix approprié de fenêtres dans l'échelle des énergies permet de sélectionner les différents isotopes du radon ou de leurs descendants émetteurs alpha, par l'énergie caractéristique des particules alpha qu'ils émettent. Dans le cas où le détecteur est placé face à un filtre ayant collecté des descendants du radon, un traitement mathématique des comptages obtenus dans chaque fenêtre d'énergie permet le calcul de l'énergie alpha potentielle volumique. C'est le principe utilisé dans les appareils de mesure rapide de l'énergie alpha potentielle (type MIMIL II H). Dans le cas où le détecteur est placé face à un volume de détection, la mesure des isotopes du radon est alors possible (cas des appareils du type Radhome).

\section{APPLICATIONS}

\subsection{Mesure des sources de radon}

Dans le cas des habitations, la source principale de radon vient du sol, une source généralement secondaire étant constituée par les matériaux de construction, deux techniques peuvent être utilisées:

- chambre d'ionisation à circulation, à travers laquelle on fait passer l'air ayant collecté le radon sortant du sol ou des murs dans un volume placé sur le sol ou appliqué sur les murs;

- appareil type BARASOL S; cet appareil, identique dans son principe aux appareils du type Radhome et utilisant la spectrométrie alpha dans un volume connu de détection, permet de mesurer, une fois placé dans le sol ou à sa surface, le flux de radon traversant la couche de sol concernée. 
Ces matériels peuvent être utilisés, soit pour la prévision du risque dans le cas des maisons à construire, soit pour constater l'efficacité d'une contremesure visant à limiter les expositions.

\subsection{Mesure des ambiances}

\subsubsection{Caractéristiques des ambiances dans les habitations}

La mesure caractérisant au mieux l'ambiance radon d'une habitation est une mesure de l'énergie alpha potentielle volumique des descendants du radon. A défaut, une mesure de la concentration du radon peut suffire pour les niveaux d'énergie alpha potentielle inférieure à $1 \mu \mathrm{J} . \mathrm{m}^{-3}$ (ce qui correspond à une concentration en radon de l'ordre de $400 \mathrm{~Bq} \cdot \mathrm{m}^{-3}$ ). L'intérêt d'une telle mesure d'ambiance réside dans l'obtention d'un résultat à la fois de précision suffisante et d'obtention rapide, représentant une situation standard et reproductible de l'ambiance étudiée.

Les techniques permettant d'obtenir ce résultat sont, dans un ordre d'intérêt croissant:

- détecteur solide de traces (achat local, durée d'exposition toujours supérieure à une semaine, résultat envoyé par un laboratoire spécialisé),

- spectrométrie gamma sur cartouche de charbon actif (achat local, envoi par la poste, exposition de quelques jours, résultats envoyés par un laboratoire spécialisé),

- mesure à l'aide de fioles scintillantes (faite par un technicien radon, rapide, mais un peu limitée en sensibilité),

- appareil intégrateur électronique (type RADHOME) utilisant la spectrométrie alpha (location locale ou envoi par poste, résultat obtenu sur place exprimé en concentration radon $\left(\mathrm{Bq} \cdot \mathrm{m}^{-3}\right)$ à $\pm 20 \%$ en quelques heures),

- appareil de mesure rapide de l'énergie alpha potentielle volumique (type MIMIL MEV $\alpha 100$, résultat exprimé en énergie alpha potentielle volumique $\left(\mu \mathrm{J} \cdot \mathrm{m}^{-3}\right)$, durée de la mesure $>1 / 2 \mathrm{~h}$, utilisation par du personnel spécialisé).

\subsection{Mesure de l'exposition individuelle}

Lorsque l'on parle de dosimétrie, on sous-entend ici "exposimétrie"; ce qui est recherché c'est l'exposition à l'énergie alpha potentielle volumique des descendants des isotopes 222 et 220 du radon. C'est, en effet, l'exposition des personnes à l'énergie alpha potentielle qui constitue le meilleur indicateur de risque. Une dosimétrie basée sur le radon total $(222+220)$, sans connaissance du facteur d'équilibre, donnera une valeur entachée d'une incertitude pouvant aller jusqu'à $300 \%$ sur le coefficient de risque.

Le seul appareil sur le marché répondant aux critères de dosimétrie individuelle est le dosimétre individuel intégré (DII) utilisé actuellement par les mineurs d'uranium (notamment tous ceux de la Cogema et de Total Compagnie Minière France). Cependant, si le port d'un tel appareil fait partie des 
contraintes de travail acceptées par un mineur, il serait peu réaliste, sauf dans des cas tout à fait particuliers, de vouloir faire porter cet appareil aux personnes du public.

II existe, par contre, une version dosimétre de site du DII qui peut permettre une approche plus souple en réalisant une dosimétrie d'ambiance dans l'habitation tout en tenant compte des temps réels d'occupation. L'appareil peut, par exemple, être placé dans la chambre à coucher et activé pendant les seules périodes d'occupation de celle-ci, les expositions diurnes n'étant pas comptabilisées. C'est la nuit que les niveaux d'énergie alpha potentielle sont les plus élevés et c'est dans la chambre à coucher que les personnes passent le plus de temps. C'est donc bien dans la chambre à coucher que les expositions des personnes seront les plus élevées. Pour que la mesure soit significative, elle doit se prolonger au moins sur un mois, trois si possible, l'idéal étant l'année.

A défaut d'utiliser ce matériel, il existe d'autres méthodes utilisant les détecteurs de traces, les cartouches de charbon actif ou bien des appareils de mesure en continu du radon ou de l'énergie alpha potentielle volumique; outre les lourdeurs et le coût des deux dernières solutions aucune de ces méthodes ne tient compte du temps d'occupation des locaux, ce qui conduit à mesurer non pas l'exposition des personnes mais celle de la maison elle-même. 